\title{
L'anthropologie micronésienne et son enseignement en Espagne
}

Teresa Del Valle

\section{(2) OpenEdition \\ 12 Journals}

Édition électronique

URL : http://journals.openedition.org/jso/1722

DOI : 10.4000/jso. 1722

ISSN : $1760-7256$

Éditeur

Société des océanistes

\section{Édition imprimée}

Date de publication : 1 juin 2001

Pagination : 81-89

ISSN : 0300-953x

\section{Référence électronique}

Teresa Del Valle, «L'anthropologie micronésienne et son enseignement en Espagne », Journal de la Société des Océanistes [En ligne], 112 | Année 2001-1, mis en ligne le 28 mai 2008, consulté le 30 avril 2019. URL : http://journals.openedition.org/jso/1722 ; DOI : 10.4000/jso.1722

\section{(c) Tous droits réservés}




\section{L'anthropologie micronésienne et son enseignement en Espagne}

par

Teresa DEL VALLE *

Cet article aborde quelques questions clefs à propos du rôle joué par l'ethnographie micronésienne dans l'enseignement et dans la poursuite de la recherche au sein de l'université du Pays basque. Dans le contexte espagnol, la recherche et l'enseignement de l'Océanie en général et de la Micronésie en particulier relèvent du domaine de l'inconnu et de l'exotique. Néanmoins, et en dépit de cette ignorance concernant un champ de recherche aussi important, je vais débattre ici des accomplissements théoriques, méthodologiques et ethnographiques réalisés dans ce domaine et montrer combien ils représentent une vraie gageure qui, somme toute, contribuera au développement de l'anthropologie en Espagne. Je vais commencer par poser quelques problèmes qui me concernent et qui sont liés au besoin (i) de contextualiser et d'articuler des champs de recherche situés à l'opposé l'un de l'autre, l'un relevant de l'Espagne, l'autre de l'étranger; (ii) d'identifier des problèmes qui transcendent les points de vue locaux et les expériences individuelles. En résumé, je vois se dessiner, à travers ce processus de compréhension de l'autre, qui s'appuie sur le concept et les expériences liés à la distance, des stratégies méthodologiques qui relèvent du fondement même de la connaissance anthropologique. Cela suppose que la connaissance des autres cultures doit être liée aux expériences, aux questions et à la connaissance que les gens en ont. En d'autres termes, nous devons contextualiser la connaissance que nous partageons avec nos étudiants afin de la rendre plus significative, surtout lorsqu'il existe un grand écart entre les cultures étudiées et leurs propres références culturelles.

\section{L'expérience coloniale et le développement de l'anthropologie à l'origine d'une méconnaissance}

La connaissance européenne des pays localisés en Asie, en Afrique et en Océanie est souvent liée à l'expérience de la dominance coloniale. $\mathrm{La}$ critique anthropologique a mis l'accent sur la relation entre l'expérience coloniale d'un pays et son impact pour le développement de l'anthropologie. Souvent, l'anthropologie, à travers sa capacité à comprendre la complexité des processus sociaux et culturels locaux, a servi à asseoir le pouvoir colonial passé ; ces processus révèlent le côté obscur de l'histoire de la discipline. Dans le cas de l'Espagne, l'expérience coloniale en Micronésie n'a pas conduit au développement de la recherche dans cette région. Les colonies ont été perdues en 1898, bien avant que l'anthropologie puisse être considérée comme une discipline scientifique pouvant aider le gouvernement à contrôler les colonies en question. Les caractéristiques de l'emprise coloniale en Micronésie, son absence de succès et sa fin brutale en 1898, suite à la guerre entre l'Espagne et l'Amérique qui ouvre sur une colonisation américaine intense, expliquent en partie l'ignorance que les Espagnols ont du Pacifique. Il est aussi important d'explorer le contexte anthropologique de l'État espagnol pendant les trois dernières décennies (1970-2000) pour comprendre le peu d'inté-

* Professeur d'anthropologie à l'université du Pays basque.

Je tiens à remercier Paul Rainbird qui, lors du colloque, m'a fait part de données concernant le cas de Angel Santos. 
rêt porté sur les cultures océaniennes au niveau universitaire.

L'index thématique d'une publication récente sur la recherche, les chercheurs et les publications anthropologiques de l'État espagnol entre 1954 et 1998, mentionne seulement trois personnes sous le titre Océanie (Prat, 1999 : 536). Pour comprendre ce manque d'intérêt, j'offre ici un résumé des principales tendances du développement de l'anthropologie dans l'État d'Espagne, à travers le rôle joué par quatre personnalités influentes. Trois de ces chercheurs : Julio Caro Baroja et Joxemiel de Barandiaran (École historique de Vienne), ainsi que Carmelo Lisón (Oxford), sont rattachés à des Écoles européennes, ce qui les a conduit à mener des travaux en Europe, essentiellement dans la péninsule ibérique. Dans le cas de Caro Baroja, sa recherche en dehors de la péninsule ibérique l'a conduit en Afrique du Nord (del Valle, 2000). La situation politique en Espagne a forcé Claudio Esteva Fabregat, la quatrième personnalité influente, à s'exiler au Mexique où il étudia l'anthropologie. Son centre d'intérêt porte sur les processus de constitution de l'identité, en particulier au sein des populations indigènes.

En dépit du passé colonial de l'Espagne en Micronésie (nous y reviendrons), la Micronésie a été une entité politique fermée depuis la fin de la Seconde Guerre mondiale, et plus précisément depuis 1947, date à laquelle les Nations-Unis ont déclaré que la Micronésie formait un Trust Territory, avec pour mandataire les États-Unis. Après quoi, il était difficile pour les citoyens non américains de mener un terrain en Micronésie. De fait, depuis et jusque dans les années 1990, la majorité des études conduites en Micronésie furent exécutées par des chercheurs américains. Ces études furent publiées en anglais et les résultats présentés au cours des enseignements donnés dans les universités américaines.

Si l'on tient compte de l'isolement qu'a connu l'Espagne suite au régime dictatorial de Franco, qui prit fin avec sa mort en 1975, et du fait que l'anthropologie en Espagne fut menée par les quatre personnes mentionnées précédemment jusque dans les années 1980, il est compréhensible que les références des travaux portant sur l'Océanie en général et sur la Micronésie en particulier fassent défaut. Nous devons penser que les résultats de la recherche anthropologique sur la Micronésie n'ont pas été introduits en Espagne avant la décennie des années 1980. Dès mon retour, en 1980, j'ai commencé à parler de la Micronésie dans mes cours d'introduction à l'anthropologie à l'université du Pays basque. J'ai présenté ma première communication, basée sur ma recherche pour la thèse $(\mathrm{PhD})$ sur Guam, à la Seconde conférence anthropologique qui s'est tenue à Madrid en 1981. Ma première publication en espagnole date de 1983. Pendant les années qui ont suivi la mort de Franco, les gens étaient davantage concernés par les questions liées à l'Europe et à leurs implications pour la compréhension et la mise en application des réformes sociales et politiques locales. $\mathrm{La}$ connaissance que l'on avait alors de la Mélanésie provenait de la lecture des travaux de Maurice Godelier, parce que beaucoup d'anthropologues espagnols s'intéressaient au marxisme et considéraient M. Godelier comme une figure dominant l'anthropologie marxiste. Plus tard, avec l'ouverture de l'Espagne sur l'extérieur et le déplacement des anthropologues vers l'Angleterre et les États-Unis, les chercheurs espagnols se sont familiarisés avec des auteurs comme Ward H. Goodenough, Marilyn Strathern, Sherry B. Ortner, Marshall D. Sahlins qui, ayant contribué très largement au champ de l'anthropologie cognitique et symbolique, du gender et des analyses historiques, mirent en exergue la richesse des ethnographies océaniennes. Enfin, certains des chercheurs découvrirent des centres d'intérêts spécifiques comme le culte du cargo à travers la littérature océanienne.

Un autre aspect important de cette analyse des raisons du peu d'intérêt porté à l'Océanie nous incite à prendre en considération le rôle joué par les anthropologues étrangers qui ont été amenés à conduire leurs travaux en Espagne, pendant les années 1970-1980, rôle important tant au niveau général que théorique. De jeunes chercheurs américains, intéressés par les nouveaux problèmes rencontrés par les sociétés contemporaines, se tournèrent vers l'Europe et choisirent la péninsule ibérique comme objet d'étude. Et même si leurs intérêts représentaient un point de vue minoritaire au sein des départements d'anthropologie des États-Unis, les travaux de Susan Tax Freeman (1970), William A. Douglass (1970), Joseph A. Aceves (1971), William A. Christian (1972), Richard A. Barrett (1974), Stanley H. Brandes (1975), Davydd J. Greenwood (1976) et David D. Gilmore (1980), parmi d'autres, montrèrent l'importance et l'intérêt qu'il y avait à travailler sur la péninsule ibérique (Prat, 1999 : 39). En conséquence de quoi, les nouveaux groupes d'anthropologues locaux, qui commencèrent leur terrain pendant les années 1975-1980, menèrent des recherches dans la péninsule ibérique, l'île de Majorque et les îles Canaries ; si bien que les publications des chercheurs étrangers et/ou locaux qui reflétaient cette zone géographique, à leur tour, influencè- 
rent l'enseignement de l'anthropologie. Plus tard, certains se rendirent dans les pays latinoaméricains et quelques autres en Afrique pour mener leur terrain. Tout cela montre combien le développement de l'anthropologie est récent en Espagne et combien les publications sur l'Océanie sont peu nombreuses. Les auteurs en sont: Teresa del Valle, Beatriz Moral, Susana Narotzky, Jose Antonio Nieto et Antonio Pérez.

Concurremment, la fin du régime de Franco fut marquée par un regain d'intérêt portant sur l'étude des communautés locales. Les anthropologues considérèrent qu'il était urgent de mener des études sur des disciplines étudiées par les folkloristes du $\mathrm{XIX}^{\mathrm{e}}$ et $\mathrm{Xx}^{\mathrm{e}}$ siècles sous l'influence $\mathrm{du}$ romantisme et de comprendre les processus de formation de l'identité sous un nouveau régime politique. Entre 1980 et 1995, la majorité des anthropologues de l'État espagnol mirent l'accent sur les études ayant pour thème l'identité au travers des rituels, des fêtes et le nationalisme de leur propre pays. En outre, il était plus facile d'être soutenu par les institutions locales en travaillant à la maison plutôt qu'à l'étranger. Quand les études en anthropologie devinrent officiellement établies dans les universités en 1995, les régions culturelles sélectionnées furent l'Afrique et l'Amérique latine. Seule l'université du Pays basque offrait un enseignement en ethnologie océanienne et des séminaires de $3^{\text {ème }}$ cycle. En raison du faible nombre de publications en anglais, l'enseignement de l'Océanie fut une expérience difficile.

En résumé, parmi les raisons qui ont fait que les connaissances et les intérêts sur l'Océanie en général et la Micronésie en particulier soient peu développés, nous citerons : l'absence d'une tradition portant sur les terrains océaniens; l'absence d'un enseignement spécifique pour le $2^{\text {ème }}$ et $3^{\text {ème }}$ cycles; le faible nombre de publications en espagnol ou autres langues locales comme l'euskara, le catalan, le gallego ou l'asturiano ; les difficultés liées à l'obtention de crédits de recherche issus d'institutions locales et publiques qui sont davantage intéressés par la recherche en Espagne ; l'absence de liens entre les universités d'Espagne, les institutions de Micronésie (université de Guam, collège de Micronésie, collège des îles Mariannes) et des États-Unis (université de Hawaii); le coût élevé des recherches en Océanie ; l'absence d'associations professionnelles à travers lesquelles des liens peuvent être établis entre chercheurs, professeurs et étudiants. À ce propos, la fondation de ESFO (European Society for Oceanists) est un pas important, mais les personnes doivent avoir mené une recherche en Océanie au préalable pour se sentir concer- nées par cette association. Il existe une association nommée Asociación española de estudios del pacifico qui publie un journal intitulé Revista española del pacifico. Cette association et son journal couvrent une large aire géographique puisqu'ils embrassent non seulement l'ensemble du Pacifique, mais aussi des pays asiatiques comme le Japon. De plus, l'emphase est mise sur l'histoire des îles et des pays qui ont fait partie de l'empire espagnol. Cette association a développé un lien étroit avec le centre de recherche de l'aire micronésienne de l'université de Guam. Néanmoins, le journal est ouvert à tous les articles portant sur des thèmes et problèmes anthropologiques.

\section{Innovation et recherche anthropologique}

En dépit du manque d'intérêt porté par l'État espagnol sur les sociétés océaniennes, nous trouvons des exemples de personnes qui se tournent vers l'Océanie pour renouveler leur approche ou leur thématique. Elles appliquent ensuite ce nouveau regard à l'étude des sociétés européennes contemporaines. L'un de ces regards concerne l'anthropologie de la sexualité. Jose Antonio Nieto estime par exemple que la prise de conscience de l'importance que constitue le domaine de la sexualité est à rechercher en Océanie, et tout particulièrement en Polynésie. D'abord, il voulut comprendre les effets de la colonisation européenne sur la sexualité et les pratiques sexuelles des Polynésiens (1993 : 216). Il fit l'effort de traduire les travaux d'auteurs importants ayant écrit sur l'homosexualité (Herdt, 1992). Dans son livre Sexualidad y deseo. Critica antropologica de la cultura, Nieto donne une bibliographie spécifique à la sexualité en Polynésie (id: 219227) avec des entrées concernant des publications datant de 1839 à 1987.

Le point de départ de Nieto est une critique sur le fait que l'anthropologie ait ignoré le domaine de la sexualité, comme si celui-ci ne relevait pas de l'expérience humaine. Il considère qu'il est donc temps que la diversité de la sexualité soit présentée pour faire pendant à l'idéologie dominante sur l'hétérosexualité ; qu'il est temps aussi de questionner les valeurs morales qui sont basées sur une compréhension de la sexualité influencée par la foi chrétienne. Selon l'auteur, les valeurs morales chrétiennes constituent un nouveau domaine de recherche.

L'approche prise par Nieto est à la confluence de différentes influences: L'histoire de la sexualité de Michel Foucault, l'anthropologie postmoderne avec une emphase sur la subjectivité de l'objet et des chercheurs, enfin sa grande 
connaissance des sources polynésiennes. Nieto peut être considéré comme une exception si on le compare aux tendances générales des anthropologues de l'État espagnol. Il a obtenu sa thèse $(\mathrm{PhD})$ à The School for Social Research de New York et a passé huit mois en 1986 à l'université de Hawaii, au Center for Sex and Society.

Une autre exception parmi la jeune génération d'anthropologues de l'État espagnol est Beatriz Moral qui obtint sa thèse à l'université du Pays basque. Moral a lié son intérêt pour le gender à la construction de la différence au sein de la société contemporaine de Chuuk. Elle contribue à la compréhension de la sexualité, de l'identité du groupe et du contrôle social dans la mesure où elle présente les conditions pour le moins paradoxales qu'une femme vit en tant que femme et en tant que sœur. Ce paradoxe est à comprendre à travers le tabou de l'inceste, tel qu'il est pratiqué à Chuuk. L'étude de Moral, tout comme ses autres articles publiés récemment $(1996,2000)$ sont d'excellentes sources que les étudiants de l'université du Pays basque lisent et discutent, non seulement lorsqu'ils suivent l'enseignement sur la Micronésie, mais aussi pendant les cours sur le gender, la parenté et la théorie féministe. De plus, les deux films documentaires qu'elle a produit avec deux professionnels du multimédia sont très appréciés des étudiants. La recherche de Moral est une source d'inspiration pour les étudiants locaux, parce que les analyses et les systèmes du gender constituent un champ de spécialisation de l'anthropologie de l'université basque. La recherche de Moral leur offre la richesse des analyses comparatives. Nous pensons que sa présence pendant deux ans (2000-2002) à l'université du Pays basque va être décisive pour éveiller l'intérêt des étudiants ${ }^{1}$.

\section{L'importance de la mise en contexte de la connaissance pour une meilleure appréciation de la part de l'étudiant européen}

Les tendances actuelles de l'anthropologie font ressortir le processus qui s'opère quand l'anthropologue entreprend sa propre recherche. Il y a un dialogue entre les gens étudiés et la personne qui les étudie. En enseignant les cultures micronésiennes, je suis consciente du besoin d'établir des liens entre les cultures étudiées et les aspects de la culture avec lesquels les étudiants sont plus familiers. Souvent, les coutumes sem- blent étranges parce qu'elles sont présentées hors contexte, mais la plupart du temps, les gens ne pensent pas que leurs propres coutumes puissent paraître inhabituelles ou étranges aux yeux de quelqu'un d'autre. La meilleure approche consisterait donc à présenter la façon même dont les Micronésiens perçoivent les Occidentaux, afin que l'on réalise combien nos propres coutumes leur semblent inhabituelles.

Avec ces idées sur la nécessité de comparer les cultures et pour que mon enseignement de la Micronésie soit une expérience utile aux étudiants, je développe les thèmes suivants: le mythe de la découverte, l'impact de la colonisation, l'importance de l'environnement pour la compréhension de l'utilisation de la terre comme de la tenure foncière et leur impact sur l'identité locale, la richesse d'un système complexe bilatéral, les mécanismes d'adoption, la complexité des systèmes de gender et des relations de gender. Je me « déplace » sans cesse entre l'ethnographie micronésienne et les exemples basques, ou à travers des études de cas, puisés au sein des communautés de la péninsule ibérique, que les étudiants connaissent bien.

Si l'on suit une approche chronologique de l'expérience coloniale de l'Espagne en Micronésie, cela nous amène au $\mathrm{XVI}^{\mathrm{e}}$ siècle pour les îles Mariannes et au XIX ${ }^{\mathrm{e}}$ siècle pour les îles Carolines. Cela commence - comme l'indiquent les manuels d'histoire - avec l'arrivée de Ferdinand Magellan à Umatac, un village côtier situé dans la partie sud de Guam, aux îles Mariannes. Prenant cet événement comme point de départ, nous trouvons que le "mythe de la découverte" est déjà bien établi. C'est parce que j'ai mené mon terrain à Umatac que je peux établir un lien entre l'interprétation historique du passé et le rôle de l'anthropologie pour l'interprétation du passé et du présent.

Si nous pensons que les institutions dominantes sont responsables de l'intérêt qu'a eu l'Espagne pour la Micronésie, nous trouvons des situations coloniales similaires en Amérique latine, où l'Église et l'État ont été des protagonistes coloniaux importants. Cela est important pour le développement des études sur le rôle de la chrétienté en Micronésie qui devraient combiner des approches historiques et anthropologiques comme des analyses du gender. À l'une des assemblées de ESFO de Bâle, Maurice Godelier a bien montré l'importance d'une étude du rôle de la chrétienté en Océanie. Et, sous son influence,

1. Beatriz Moral a reçu une bourse post-doctorale du gouvernement basque, afin de conduire une recherche sur la culture matérielle de Micronésie dans les institutions européennes. Elle sera au département de Philosophy of Values and Social Anthropology de l'université du Pays basque. Le Second colloque international sur la Micronésie a eu lieu à cette université au printemps 2001 
deux conférences ont eu lieu sur ce thème à Paris en 1999 et 2000. Suite à l'initiative de Godelier, j'ai présenté un programme de recherche sur l'activité missionnaire catholique $\mathrm{du} \mathrm{xx}^{\mathrm{e}}$ siècle en Micronésie à l'une des sessions du Premier colloque international sur la Micronésie qui s'est tenu à Marseille. Cette communication s'intitulait : « The missionary activity in Micronesia : a bridge between cultures» (l'activité missionnaire en Micronésie : un pont entre deux cultures). J'y ai abordé deux thèmes : 1) l'importance des relations de gender et des systèmes de gender pour la compréhension du prestige et du pouvoir (par exemple : les modèles, les rôles des missionnaires, la distribution des tâches) ; 2) le besoin de mettre en contexte les activités missionnaires qui relèvent du gender dans un contexte social, historique et politique, tant en Micronésie que dans les pays d'où est originaire l'activité missionnaire.

\section{Le mythe de la découverte et son impact}

Le premier contact entre les Occidentaux et les habitants des îles Mariannes remonte à l'an 1531, quand Ferdinand Magellan toucha terre à Guam. Afin de questionner l'idée que les Chamorro furent découverts par les Européens, nous avons analysé le carnet de voyage d'Antonio Pigafetta (1963:63-70), une source remarquable en ce qui concerne les contacts. Pigafetta écrivit :

« Les gens des îles des Voleurs furent si surpris de nous voir que nous avons pensé que jusqu'à ce jour, ils n'avaient vu personne d'autres que les gens de leurs propres îles » $\left(i b .:\right.$ : 70) ${ }^{2}$.

Nous n'avons aucune source écrite sur l'interprétation que les Chamorro firent de ces visiteurs. Néanmoins, nous pouvons nous reposer sur les grandes compétences de la navigation qu'avaient les Chamorro et sur les contacts qu'ils entretenaient avec les habitants des autres îles, en particulier avec les îles Carolines. Même si, après la conquête espagnole, le commerce avec les îles Carolines devint discontinu, des informations ont été recueillies oralement et documentées au début $\mathrm{du} \mathrm{XIX}^{\mathrm{e}}$ siècle. Pour attester de l'existence de ce système d'échange aux temps anciens, on peut s'appuyer sur le fait que quand le commerce entre les Carolines de l'ouest et les Mariannes fut réétabli en 1788, le groupe des habitants des îles Carolines qui naviguèrent vers Guam utilisèrent la route des étoiles, un savoir qui était préservé dans les chants.
Une autre preuve de l'existence de ce système d'échange inter-îles à Guam est la présence de pirogues très rapides qui firent l'admiration de Pigafetta et de ses compagnons à une date aussi ancienne que 1521 . La vitesse de ces pirogues atteignait neuf milles par heure par vent faible et quinze milles par heure par vent fort. Les Chamorro possédaient donc bien le type de pirogues leur permettant de participer aux échanges sur de grandes distances.

Magellan fut suivi par Miguel de Legazpi qui, en 1565, sur le chemin des Philippines, clama officiellement que toutes ces îles devaient être placées sous tutelle de la Couronne espagnole. Cette revendication prit effet en 1668, quand le droit espagnol fut établi avec la venue des soldats et l'ouverture d'un premier poste missionnaire par les Jésuites. Cette date ancienne de l'introduction de la chrétienté est importante parce qu'elle permet de mieux comprendre le fort impact de cette religion aux îles Mariannes au cours des siècles suivants.

Comme souvent au cours des premières rencontres entre les visiteurs coloniaux et la population locale, l'expérience Chamorro - Espagnols n'était pas très intéressante. La mise en application d'un règlement étranger donna lieu à des conflits armés de 1671 à 1695 entre la population indigène et les soldats espagnols. Le déplacement des Chamorro de Saipan et Tinian sur Guam s'ensuivit. Il facilita le contrôle espagnol d'une population décimée par les guerres et les épidémies. Au bout du compte, la population des îles Mariannes, estimée à 30000 âmes à l'époque des premiers contacts en 1668 , ne comptait pas plus de 5000 personnes en 1699. À l'exception de quelques centaines de réfugiés qui s'étaient cachés sur l'île de Rota, le reste de la population résidait sur Guam.

Je me sers de la connaissance que l'on a de l'ancienne société des Chamorro pour introduire les étudiants aux caractéristiques des sociétés insulaires et développer la relation qu'ils nouent avec l'environnement physique, leur organisation sociale et leurs systèmes de croyances. Je traite des Chamorro comme d'une étude de cas pour expliquer la relation que ces gens ont avec leur environnement, les savoirs tels qu'ils se manifestent à travers la façon dont ils organisent leurs habitats, leurs choix des sites pour des villages côtiers et à l'intérieur des terres. J'utilise des diapositives de la Maison de Taga, situé sur l'île de Tinian, de manière à ce qu'on puisse juger visuellement de leurs techniques de construction. Je montre combien l'habitat reflète la pra- 
tique d'une économie de subsistance diversifiée basée sur l'horticulture, la pêche et le commerce. Je propose ensuite une analyse des sites préhistoriques : dolmens et cromlechs du Pays basque en relation à leur environnement, que je compare aux sites océaniens. Enfin, je discute de la chronologie des sites océaniens et des sites basques de façon à établir des parallèles entre l'habitat océanien et l'histoire européenne.

Quand je parle des premiers contacts entre Européens et Chamorro, je ressens le besoin de mettre en contexte cette expérience coloniale dans un cadre plus large, qui est celui du colonialisme du Nouveau Monde. Les îles Mariannes n'ont jamais été une colonie importante pour l'Espagne. Il est donc nécessaire d'expliquer les intérêts coloniaux en jeu dans ce Nouveau Monde que sont les Philippines et montrer que les Mariannes n'étaient qu'un simple lieu à partir duquel les Espagnols pouvaient exercer des revendications militaires dans cette partie du monde. Je suis aussi confrontée à un manque d'information concernant la population locale, ce qui n'est pas le cas pour le reste des communautés micronésiennes qui, elles aussi, ont été sous influence coloniale japonaise, espagnole, allemande, américaine. Aux îles Mariannes, les siècles de domination et la diminution de la population chamorro sont à l'origine du fossé entre le passé lointain et le présent.

Je m'intéresse aussi à deux types d'analyses : l'une est comparative et prend en compte le fait que la culture basque est la référence de la majorité des étudiants de l'université du Pays basque ; l'autre est méthodologique.

Toute personne intéressée par la culture basque connaît l'importance que l'on donne aux origines méconnues de la langue et à ses différentes théories. Les sites archéologiques indiquent que l'homme s'est installé dans cette région du monde dès $20000 \mathrm{BC}$. La période du néolithique est, quant à elle, bien représentée par les cromlechs et les dolmens. Cela me permet d'opposer la situation basque au cas des Chamorro. En effet, le peu de connaissance que nous avons de l'ancienne société chamorro repose sur des croquis de voyages, des récits de missionnaires, des prospections archéologiques et des interprétations du passé basées sur des légendes et des chants.

Cela m'amène à faire une analyse des différents types de sources et à les évaluer. J'explore alors le concept d'ethnohistoire et je développe la méthodologie requise par une situation de ce genre, où la connaissance locale du passé a été quasiment perdue. Je mets aussi l'accent sur l'importance d'une analyse des silences ou des vides concernant l'ancienne société chamorro, qu'il n'est plus possible de combler. Les étudiants peuvent émettre leurs propres suggestions sur l'ancienne société chamorro, ce qui les amène à mieux comprendre les types de problèmes auxquels nous sommes confrontés. Je rappelle aussi que des données ethnographiques peuvent être obtenues en recourant aux sources conservées aux Archivo de Indias de Séville, aux archives de Madrid, Loyola, Bérriz, et aux quelques documents officiels.

Si l'on tient compte du fait que les données que nous avons concernant l'ancienne société chamorro embrassent les thèmes suivants : organisation sociale, tenure foncière, utilisation des terres, croyances, navigation, pratiques sexuelles et connaissance de l'environnement, seule une approche holistique pourrait aider à la compréhension de la société. Après avoir opportunément présenté le cadre général des sociétés micronésiennes et parler de certaines de leurs caractéristiques, je peux ensuite me concentrer sur certains aspects, entre autres ceux liés à la parenté, à l'usage de la terre et à la tenure foncière.

\section{Une approche comparative de la parenté et de l'organisation sociale micronésienne}

En donnant un cours général de haut niveau sur la parenté et l'organisation sociale, j'établis un parallèle entre la théorie et les exemples ethnographiques qui ont été discutés préalablement, en insistant sur la parenté et l'organisation sociale. Je prends pour exemple les Chamorro parce qu'ils offrent un exemple très intéressant de bilatéralisme. La société chamorro est l'exemple même du fonctionnement d'une société bilatérale où les liens de parenté sont constamment actualisés avec une profondeur généalogique de deux générations. C'est un cas type qui montre combien les rituels du cycle de la vie réactivent les liens de parenté et sont indissociables d'une forte idéologie de l'endogamie géographique. L'endogamie, telle qu'elle se pratique lors du mariage, à l'intérieur comme à l'extérieur du village de Umatac, est au fondement de l'identité.

J'insiste sur la grande flexibilité du système bilatéral. Il offre un large éventail d'options pour les hommes et pour les femmes. Nous discutons ces options en adoptant le point de vue de l'anthropologie féministe et comparons ce système à la matrilinéarité et à la patrilinéarité que nous trouvons au sein des sociétés micronésien- 
nes. La société chamorro insiste sur la flexibilité dans la façon dont une personne réactualise ses droits et ses obligations après le mariage ou, dans le cas des femmes non mariées, après qu'elles aient eu leur premier enfant. Cet aspect est utile aux discussions portant sur la maternité, parce qu'avoir un enfant peut être plus pertinent que d'être marié. Cette définition de la maternité sociale ouvre sur l'adoption qui, abordée de manière comparative, permet de comprendre comment elle s'exprime et comment elle est vécue par les sociétés micronésiennes et par les différentes communautés de la péninsule ibérique.

Un second aspect que les étudiants apprécient est la richesse de la société chamorro quant à l'importance des rituels liés au cycle de vie d'un individu. J'attire l'attention sur la participation des hommes et des femmes, mais aussi sur la plus grande complexité de la participation des femmes, puisqu'elles doivent répondre à une double obligation, tant du côté de leur père et de leur mère que du côté du père et de la mère de leur mari. Puis, nous discutons de la relation qui existe entre la parenté et la terre. Même si les femmes, tout comme les hommes, héritent de la terre, les hommes deviennent davantage propriétaires terriens que les femmes. J'analyse des situations idéales en adoptant le parti de la femme comme un exercice de méthodologie féministe. Idéalement, la situation où les femmes sont privilégiées va de pair avec une terre indivise, lorsque les prétendants légaux ont potentiellement le droit de se référer à leur propre terre ou de la nommer terre de famille. C'est dans ce contexte de relations de gender que la position de la femme doit être appréhendée.

\section{Le jeu entre la terre, la résidence et les rituels}

Comme je l'ai déjà écrit dans un article précédent (del Valle, 1992), la célébration des rituels liés au cycle de vie d'un individu, qui a lieu dans des parcelles de terres bien spécifiques, établit un lien étroit avec le passé, ce qui, à son tour, renforce les liens de parenté et l'identité de la communauté. Les rituels liés au cycle de vie se conforment à une structure type, où les liens sociaux sont actualisés, exprimés et transmis. La permission d'utiliser des espaces supplémentaires, autre que celui occupé par la maison de famille, doit être négociée avec les voisins. Cette négociation est particulièrement intense durant les deuils parce que, pendant ce rituel, la coutume désigne des espaces bien définis pour chaque activité, afin que le rituel puisse être mené à bien. La proximité résidentielle des membres de la parentèle est importante pour obtenir la permission d'utiliser les terres des proches voisins. La présence constante de proches parents pendant ces rituels et leurs charges émotives, économiques et sociales mettent en exergue l'étroitesse des relations entre les individus et compensent l'absence de généalogie. Ces rituels servent bien à renforcer les liens sociaux, comme à visualiser le grand nombre de parents proches d'Ego.

Ces exemples, puisés dans la société chamorro, sont importants puisqu'ils aident à la compréhension des relations existant au sein de l'habitat et de la maisonnée, comme à la compréhension de la position des hommes et des femmes dans une société contemporaine. J'analyse ainsi, avec les étudiants, des cas similaires rencontrés auprès de la société basque contemporaine et dans d'autres communautés de la péninsule ibérique. Après quoi, nous évaluons les règles de résidence pour mettre en avant les équités ou les inégalités des relations de gender.

La société basque est toujours concernée par l'identité. Dans le cas des Chamorro, j'insiste sur les différents moyens que les gens utilisent pour le développement et la reconnaissance de l'identité. Les Basques, tout comme les Chamorro, donnent de l'importance à la terre, à la langue et à l'organisation sociale, trois éléments constitutifs de l'identité.

\section{Subversion et groupes silencieux}

Je vais établir quelques parallèles entre la stratégie de subversion développée par les Chamorro et celle développée par d'autres gens en situation marginale. Dans un article précédent, j'ai développé le concept de subversion et introduit celui de " groupes silencieux » développés par Edwin Ardener (1975). Je mentionne également les contributions de l'anthropologue Dolores Juliano (1992) qui analyse les stratégies développées par des groupes de femmes marginales pour exprimer leur pouvoir, leurs modèles et leurs alternatives pour subvertir les coutumes et les valeurs.

Les Chamorro ont bien conscience du fait que leurs îles ont été occupées par différentes puissances et que leurs terres ont été sujettes à des négociations et des législations établies par l'Espagne, le Japon et les États-Unis. Comme nous l'avons vu précédemment, l'histoire du colonialisme remonte à l'an 1521 et, en dépit d'une reconnaissance de droits locaux au $\mathrm{Xx}^{\mathrm{e}}$ siècle, leur histoire est bien celle d'une imposition qui vient du dehors. Il y a peu de données concernant le système de tenure des terres avant 
la colonisation espagnole; et quand nous évoquons la période moderne, la grande majorité des registres fonciers remontent aux années 1850. Dans un système bilatéral, comme celui des Chamorro, les gens ne font pas référence à des ancêtres. Dans la plupart des cas, lorsque les gens retracent l'histoire de leurs terres, ils se réfèrent à la seconde génération ascendante. Pour exprimer une occupation continue, ils emploient l'expression de « terre de famille » ou de terre appartenant à la famille «depuis très très longtemps ». Cette proposition peut aussi être utilisée pour exprimer le fait que la coutume a de profondes racines. En 1975 et 1976, je constatais :

« La connaissance relative aux origines des gens qui ont des droits sur la terre, ou des gens qui revendiquent une terre, remontaient à la deuxième génération ascendante $(\mathrm{G}+2)$. Par exemple, un individu (Ego) disait qu'une certaine parcelle avait été originellement détenue par son grand-père (FF d'Ego). Si j'avais demandé à Ego d'autres informations généalogiques, sa réponse aurait été qu'il supposait que le père de son père $(\mathrm{FF})$ avait hérité de la terre de son père (FF d'Ego) » (del Valle, 1979 : 117-18).

Dans les cas de terres indivises, les gens remontaient à la personne dont le nom apparaissait dans les registres fonciers. D'autres affirmaient que la terre de sa famille remontait au temps d'un gouverneur espagnol, mais il n'y avait aucune généalogie pour le prouver. En dépit de ce manque de données qui ne permet pas d'établir un lien historique entre le présent et le passé ancestral d'avant la colonisation, les gens ont développé des liens symboliques et y font référence comme s'ils étaient fondés sur la continuité de leurs croyances. Dans le cas des Chamorro, à Guam, le lien le plus fort avec le passé, au moins jusqu'en 1976, consistait en la croyance que certains espaces de Guam étaient habités de façon permanente par des esprits appelés taotamo'na depuis les temps anciens et jusqu'à aujourd'hui. Quand je vivais à Guam, j'ai pu constater que les croyances qui entourent ces taotamo'na était très fortes. Cette constante référence, exprimée par les gens de différents milieux, témoigne de ce qu'au niveau symbolique, la terre soit demeurée aux mains des propriétaires originaux. Je considère ce type de référence à la propriété et au passé comme une stratégie subversive. Les références au taotamo'na évoquent le pouvoir d'un passé d'autrefois et effacent toute trace d'occupation coloniale de l'île. C'est sans doute une stratégie de résistance symbolique, comme je vais le montrer avec le cas d'Angel Santos, qui a réclamé des terres autrefois privées et aujourd'hui détenues par le gouvernement. De tels actes sont rares et sont considérés comme étant extrêmement subversifs.

Angel Santos, qui a été par deux fois sénateur à Guam, décida en 1999 de réoccuper la terre de son grand-père. En septembre 1999, il écrivit au procureur général des États-Unis à Guam et au procureur nommé Janet Reno pour dire qu'il avait l'intention d'occuper la terre de son grandpère qui faisait partie des 10000 acres nommés « terres militaires en excès » en 1993. Santos commença à déboiser sa terre de famille en octobre 1999, en conséquence de quoi il fut inculpé en décembre de la même année et envoyé dans une prison fédérale de Californie pour six mois le 8 janvier 2000. Plusieurs civils et des organisations religieuses américaines à Guam, Hawaii, dans l'Ohio et au Japon ont soutenu Angel Santos. Il est intéressant de mentionner que dans la résolution adoptée par le Conseil de l'Église du Christ Uni pour les minorités raciales et ethniques, on peut trouver la phrase suivante : «il suivait une autorité spirituelle supérieure quand il réclama ses terres ancestrales ». Il serait intéressant de voir si le cas de Santos va provoquer d'autres revendications et d'autres actions parmi la population chamorro ou bien si ce fut un événement isolé.

Les espaces où les gens associent la terre aux taotamo'na sont des lieux où l'on trouve les vestiges des piliers de pierre (de maison) appelés latte, ou encore certains espaces forestiers. Pendant mon séjour à Umatac et à différentes reprises, des histoires à propos de terres occupées par ces esprits m'ont été racontées. En fait, dans la maison où je logeais pendant mon terrain, l'aire comportait des latte. La femme de la maison nettoyait et plantait cette aire, mais les autres membres de la famille évitaient l'endroit. Ils avaient peur de ce lieu habité par les esprits des ancêtres. Ces esprits habitaient le milieu de la forêt, dans des espaces dont les droits de propriété n'étaient pas clairs, des lieux privés que le gouvernement s'était appropriés autrefois. Dans les narrations recueillies, les femmes étaient les seules à avoir été attaquées par les taotamo'na alors que les hommes étaient ceux qui demandaient pardon aux esprits.

Une femme m'a raconté que les gens avaient peur des pierres qui se trouvaient dans son jardin. Quand la maison fut construite, un homme vint donner un coup de main pour la construction, mais ne revint jamais plus, parce qu'il avait eu peur des pierres. Plus tard, le mari de cette femme me confirma que ces pierres étaient bien des latte. Mais la femme n'avait pas peur de ces 
pierres, elle plantait des fleurs aux alentours et appelait cet espace son « jardin de pierres». Néanmoins, certaines personnes âgées ne vinrent jamais dans cette maison parce qu'ils avaient peur des taotamo'na.

\section{Conclusion}

Après avoir développé les raisons de l'existence, en Espagne, d'un savoir limité concernant l'Océanie de manière générale, et plus particulièrement la Micronésie, j'ai attiré l'attention sur le besoin d'établir des relations entre l'ethnographie de l'autre, que l'on trouve dans les sociétés micronésiennes, et l'ethnographie de l'autre auquel les étudiants peuvent faire référence. Il est évident que l'anthropologie est d'une richesse exceptionnelle quand la discipline adopte une démarche comparative. Les éléments qui apparaissent dans une culture peuvent conduire à des questions très différentes quand elles sont mises hors contexte. L'un des défis que l'anthropologie offre est sa dimension comparative et cela peut s'exprimer à travers la connaissance que nous donnons des différents cas de figures issus de sociétés diverses. Mais il est important de contextualiser la connaissance que nous donnons, de manière à ce que les étudiants puissent faire des parallèles entre leurs propres connaissances et expériences et la connaissance que nous donnons d'une autre culture. Enseigner l'Océanie, et la Micronésie en particulier, nécessite d'établir des liens entre des situations, non seulement différentes, mais aussi avec des gens qui sont éloignés. C'est pourquoi nous devons notamment tenir compte des manières de raconter les expériences historiques et les différences environnementales... Nous pouvons aussi créer des liens à des niveaux plus généraux, par exemple, sur la quête de l'identité, sur l'importance donnée aux liens familiaux, sur les stratégies que les gens développent dans des situations de silence et de domination, etc. L'approche anthropologique qui met l'accent sur le besoin de voir les choses et d'appréhender la réalité à travers le point de vue d'un natif doit aussi être appliqué à l'enseignement.

\section{BIBLIOGRAPHIE}

Ardener, Edwin, 1975. Belief and the Problem of Women, in Ardener, Shhirley (ed.) Perceiving Women, London, J.M. Dent and Sons, pp. 1-17.

DEL Valle, Teresa, 1979. Social and Cultural Change in the Community of Umatac Southern Guam, Guam, Micronesian Area Research Center.

_, 1984. El funcionamiento y significado del kindred en la comunidad chamorra de Umatac, en la isla de Guam (Islas Marianas), Comentaris d'Antropologia Cultural 5, pp. 135-152.

_, 1992. Land and Ritual Linkages with the Past in Chamorro Society (Mariana Islands). Paper presented at the First European Colloquium on Pacific Studies, University of Nijmegen December 17-19.

—, 2000. Relecture des œuvres de Barandiarán et de Caro Baroja, Ethnologie française 30, pp. 293-297.

Colobrans, Jordi, Martínez Angel, Prat, Joan, 1996. Entrevista con Claudio Esteva-Fabregat", in Joan Prat and Angel Martínez (eds.) Ensayos de antropología cultural, Barcelona: Ariel, pp. 16-27.

Herd, Gilbert H. (ed.), 1992. Homosexualidad ritual en Melanesia, Madrid, Fundación UniversidadEmpresa.

Juliano, Dolores, 1992. El juego de las astucias. Mujer y construcción de modelos sociales alternativos, Madrid, Horas y horas.

Moral, Beatriz, 1996. Sobre la interesante concepción de los genitales en Chuuk (Micronesia), Revista española del Pacífico 6, pp. 111-136.

—, 1997. Conceptualización de la mujer, del cuerpo y de la sexualidad en Chuuk (Micronesia), umpublished $\mathrm{PhD}$ thesis, Universidad del País Vasco.

—, 2000. La hermana en Chuuk (Micronesia): mujer paradójica, mujer plural, in del Valle, Teresa (ed.) Perspectivas feministas desde la Antropología Social, Barcelona: Ariel, pp. 125-153.

NARotzky, Susana, ***. La herencia inalienable : Tierra, iglesia e independencia en Polinesia, in Pascual Fernández, J. (coord.). Procesos de apropiciación y gestión de recursos comunales, Tenerife, VI Congreso de Antropología, FAAEE/ACA, pp. 141155.

Pigafetta, Antonio, 1963. Primer viaje en torno del globo, Madrid, Espasa Calpe.

Nieto, José Antonio, 1993. Sexualidad y deseo. Crítica antropológica de la cultura, Madrid: Siglo XXI.

Prat Carós, Joan, 2000a. Informations génerales, Ethnologie française XXX, pp. 305-315.

-, 2000b. Après The People of the Sierra, Ethnologie française XXX, pp. 221-232.

United Church of CHrist, 2000. News release, May 25 (2 pages).

\section{Mots-clefs / Keywords}

Micronésie, Chamorro, anthropologie, enseignement, Pays basque. Micronesia, Chamorro, anthropology, teaching, Basque country. 